\title{
Short-Term Outcome of Focused Shock Wave Therapy for Sural Myofascial Pain Syndrome associated with Plantar Fasciitis: a Randomized Controlled Trial
}

\author{
E. Carlisi ${ }^{1}$, F. Manzoni ${ }^{2,3}$, G. Maestri ${ }^{1}$, L. M. R. Boschi ${ }^{1}$, C. Lisi ${ }^{1}$ \\ 1 Rehabilitation Unit, IRCCS Policlinico San Matteo Foundation, University of Pavia, Pavia, Italy \\ 2 Clinical Epidemiology and Biometric Unit, IRCCS Policlinico San Matteo Foundation, Pavia, Italy \\ 3 Health Promotion-Environmental Epidemiology Unit, Department of Hygiene and Health Prevention, Health \\ Protection Agency, Pavia, Italy
}

\section{CORRESPONDING AUTHOR:}

Ettore Carlisi

Rehabilitation Unit

IRCCS Policlinico San Matteo Foundation

piazzale Golgi 19

27100 Pavia, Italy

E-mail: e.carlisi@smatteo.pv.it

DOI:

10.32098/mltj.03.2021.17

LEVEL OF EVIDENCE: 2B

\begin{abstract}
SUMMARY
Background. This study aims to describe prevalence and clinical features of the sural myofascial pain syndrome (SMPS) in a population affected by plantar fasciitis and to investigate if a shock wave treatment extended to the gastrocnemius-soleus trigger points is more effective in speeding up the improvement of heel pain and sural myofascial pain than a standard treatment exclusively targeted at the plantar fascia.

Methods. Among 81 subjects affected by plantar fasciitis, 55 showed concomitant SMPS and were randomized to receive focused shock wave therapy for the plan$\operatorname{tar}$ fascia and for gastrocnemius-soleus trigger points or for the plantar fascia only. We monitored heel pain (FFI-A) and foot function (FFI-B) using the Foot Function Index. Pressure pain threshold (PPT) and Delta-PPT of the gastrocnemius-soleus trigger points was assessed with a digital algometer. Outcome measures were monitored in a follow-up four weeks after the treatment.

Results. The prevalence of SMPS resulted to be $67.9 \%$. At the follow-up, no statistical significance in the comparisons between groups was found for FFI-A and FFI-B total score and also for PPT and Delta-PPT values, despite comparisons within the study group (follow-up versus baseline) showed a significant reduction of FFI-A total score $(\mathrm{p}<0.001)$, FFI-B total score $(\mathrm{p}=0.029)$ and Delta-PPT values $(\mathrm{p}=0.018)$, with a consensual increase of PPT values $(\mathrm{p}=0.017)$.

Conclusions. We pointed out a high prevalence of sural trigger points in subjects affected by plantar fasciitis. In a short-term perspective, the extension of a focused shock wave treatment to the gastrocnemius-soleus trigger points resulted to be a safe but ineffective option in reducing heel pain and sural myofascial pain, if compared to a standard treatment.
\end{abstract}

\section{KEY WORDS}

Myofascial pain syndrome; myofascial trigger point; plantar fasciitis; shock wave; tendinopathy.

\section{BACKGROUND}

Myofascial pain syndromes are musculoskeletal disorders characterized by the presence of trigger points associated to local pain and stiffness. Active trigger points are classically detailed as spontaneous foci of pain, with palpable taut bands and accentuated by pressure, whereas latent trigger points as clinically quiescent foci, becoming painful only when palpated (1). Despite the diagnosis of myofascial pain syndrome is mainly based on clinical findings, measuring pressure pain threshold using algometry is important to quantitatively monitor trigger points response to therapy (2). Travell and Simons first 
described a myofascial pain syndrome in the gastrocnemius-soleus muscle complex, characterized by the presence of trigger points associated to calf cramps and to referred heel pain (1).

Plantar fasciitis is the most common cause of heel pain, especially between the ages of 40 and 60 years $(3,4)$; it is experienced in both recreational and professional athletes of different sports, particularly in runners (5). Subjects affected by plantar fasciitis often show tightness of the triceps surae, with a consequent limitation of the ankle dorsiflexion, Patel reporting an isolated contracture of the gastrocnemius in $52 \%$ in case of chronic heel pain $(6$, 7). Despite patients suffering chronic heel pain are also often affected by calf cramps, especially at night, the exact prevalence of sural myofascial pain concomitant to plantar fasciitis is actually unknown.

Among the great variety of therapies, that have been reported for the treatment of plantar fasciitis, shock wave therapy (a transient three-dimensional pressure wave characterized by a sudden increase in pressure within a few nanoseconds) is actually considered a consolidated treatment $(8,9)$. In 2015, Gollwitzer demonstrated that an optimized protocol of focused shock wave therapy is effective in the treatment of plantar fasciitis, assessing an improvement of heel pain in a follow-up at three months after the end of the therapy (8).

On the contrary, only few medical reports, at present, show the efficacy of shock wave therapy on myofascial pain syndromes (10-13). In 2014, Moghtaderi showed that treating with shock waves both plantar fascia and gastrocnemius-soleus trigger points is more effective (two months after the end of treatment) in reducing heel pain than solely treating the plantar fascia, in a population affected by plantar fasciitis with sural myofascial pain, but the outcome of shock waves on sural trigger points was not investigated (13). In a study on trapezius muscle's trigger points, Jeon showed an increase of the pressure pain threshold after the first shock wave session and at the end of treatment (11), suggesting that myofascial pain may have an earlier response to shock wave therapy than tendon pain, in case of an association between a myofascial pain syndrome and a tendinopathy.

The aim of the present study is to describe prevalence and clinical features of the sural myofascial pain syndrome in a population affected by chronic heel pain diagnosed as plantar fasciitis and to investigate if a shock wave treatment extended to the gastrocnemius-soleus trigger points is more effective in speeding up the improvement of heel pain and sural myofascial pain than a standard treatment exclusively targeted at the plantar fascia.

\section{MATERIALS AND METHODS}

\section{Study design}

This study was conducted as a randomized controlled trial with blind outcome assessors. It was registered at clinicaltrial.gov (NCT04385329), it was approved by the local ethical board (protocol number: 20160017179- date of approval: 07/04/2016) and was drawn up in accordance with the CONSORT 2010 Statement Guidelines and with the current version of the World Medical Association Declaration of Helsinki. The trial was carried out in accordance with the standards of good clinical practice and the ethical standards of the journal (14).

\section{Patients}

All consecutive subjects, affected by heel pain and referred to our medical centre from 2016 to 2018, were screened for inclusion in an outpatient rehabilitative setting. The recruitment procedure, performed by a physical and rehabilitation medicine specialized physician, included a clinical examination of the affected and contralateral lower limb, a sonographic examination of the plantar fascia and an X-ray of the affected ankle and foot.

Inclusion criteria were: adult age (18 to 75 years) and written informed consent; unilateral heel pain of four weeks or longer duration; pain to digital pressure in the insertional area of the plantar fascia at the calcaneum; sonographic examination showing a plantar fascia thickness greater than $3.8 \mathrm{~mm}$ (see procedures for details); concomitant presence of a sural myofascial pain syndrome (SMPS), diagnosed on the basis of the finding, at the physical examination, of trigger points of the gastrocnemius-soleus muscle complex, according to Travell and Simons' original description (1). List and location of the trigger points are shown in figure 1. Exclusion criteria were: corticosteroid injections or other physical therapies since the onset of the current pain episode (except pharmacological pain treatments and shock-absorbing heel pads); general contraindication to shock wave therapy (pregnancy, bleeding disorders or anticoagulant drug usage, cancer in the focal area); clinical signs of lumbar radiculopathy at physical examination; ankle osteoarthritis, diagnosed on the basis of clinical and radiographic findings; previous fractures or surgery in the affected ankle and foot; rheumatologic diseases; plantar fibromatosis.

Subjects satisfying eligibility criteria, except for the presence of SMPS, were also screened at baseline, in order to compare their demographics and heel pain features to those of the patients affected by SMPS, but they were not randomized. Enrolled patients were randomized to receive 


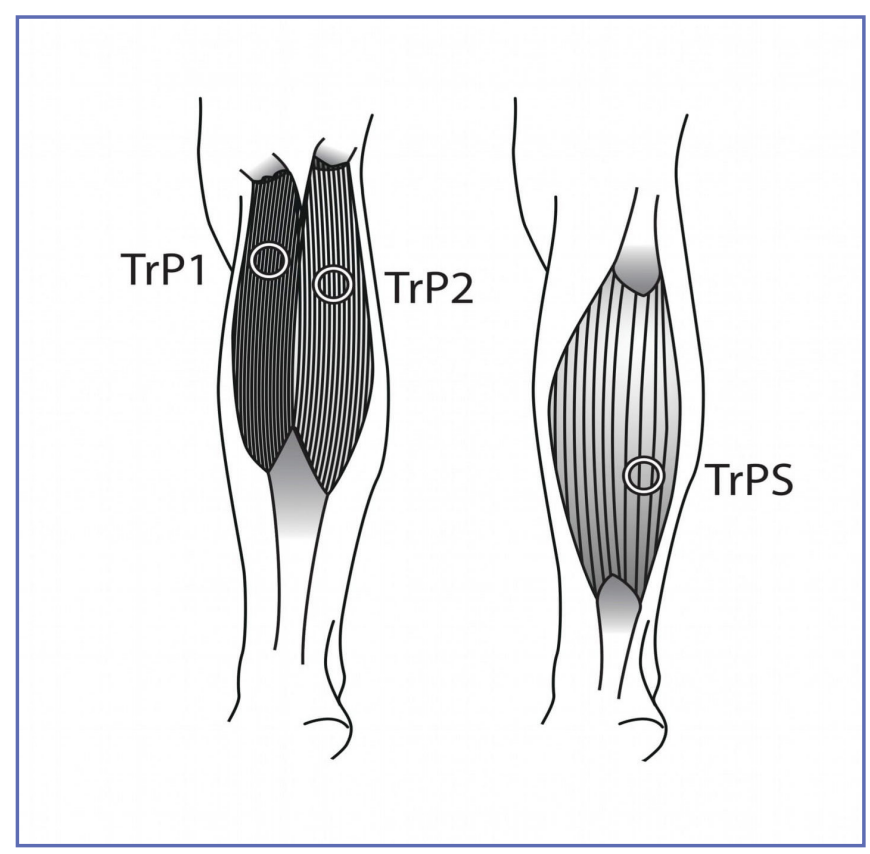

Figure 1. Location of the trigger points of the gastrocnemius-soleus muscle complex.

$\operatorname{Tr} \mathrm{P} 1$ : trigger point of the medial gastrocnemius muscle; $\operatorname{Tr} \mathrm{P} 2$ : trigger point in the lateral gastrocnemius muscle; TrPS: trigger point in the distal lateral gastrocnemius/soleus muscle.

either focused shock wave therapy to the plantar fascia and to gastrocnemius-soleus trigger points (study group) or to the plantar fascia only (control group). The patients' allocation was performed by a computer-generated randomization list, using the command "ralloc" of the STATA statistical software, and by the use of prefilled envelopes, indicating which group each patient was allocated to. A clinician blinded to the treatment allocation recorded demographics and clinical features (heel pain duration, side of the pathology, presence of heel spur, sural trigger points localization and related symptoms, sonographic thickness of the plantar fascia) and evaluated patients for outcome measures at baseline (the week prior to the treatment) and at the followup. Patients and clinicians performing treatments were not blinded to the treatment allocation.

\section{Procedures}

We monitored heel pain and foot function using the Foot Function Index, a self-administered questionnaire designed to measure foot performances in relation to symptoms. The Foot Function Index is structured in two sections of nine items each, investigating foot pain (FFI-A) and function (FFI-B) respectively. The total score of each section ranges from zero (no pain/no functional limitation) to 100 (intolerable pain/complete inability) (15). The two scores were separately analysed. Pressure pain threshold (PPT) of the gastrocnemius-soleus trigger points was assessed by means of a digital hand-held algometer (see procedures for details). $\mathrm{PPT}$ is the minimal force that induces pain and its measurement is useful to quantitatively evaluate the effect of a treatment on trigger points. Since comparing PPT measurement of abnormal painful areas with normal tissues on the opposite side of the body is probably more reliable than using reference values for specific muscles $(2,16)$, we specifically monitored variations over time of the difference between trigger points' PPT on the painful side and the PPT measured on the asymptomatic contralateral calf (Delta-PPT). Outcome measures were monitored at baseline and in a follow-up four weeks after the end of the shock wave treatment. Patients reporting a FFI-A score $\leq 40$ at the follow-up were registered as "early responders" to shock wave therapy.

As part of the recruitment procedure, the sonographic examination of the plantar fascia was performed with respect to morphology and echo-texture, in both longitudinal and transverse planes, through a linear probe (7.5$12 \mathrm{MHz}$ ) connected to an ultrasound scanner (ESAOTE MYLAB FIVE, Genova and Florence, Italy ${ }^{\circledR}$ ). We specifically measured thickness of the plantar fascia $1 \mathrm{~cm}$ away from the insertion at the calcaneum (17). According to literature, we considered a thickness more than $3.8 \mathrm{~mm}$ consistent with a status of plantar fasciitis $(18,19)$. The sonographic thickness of the contralateral plantar fascia was also recorded.

With the patient laying down in prone decubitus, trigger points were identified and marked by a palpatory examination of the calf. On the contralateral calf (asymptomatic side), we marked a specular point, in the same position as the trigger point on the painful side. We used a digital hand-held algometer, equipped with a $1 \mathrm{~cm}^{2}$ probe and with a display unit (Tracker Freedom wireless-J Tech Medical ${ }^{\circledR}$ ) to measure pressure pain threshold (PPT). Before the test, subjects were instructed to orally warn the operator when the sensation of increasing pressure was turning into pain. During the test, a perpendicular pressure was applied with the rubber tip of the hand-held algometer on the trigger points on the painful side and on the corresponding contralateral sites. We used the visual control of an electronic display to apply a linearly increasing pressure of about $1 \mathrm{~kg} / \mathrm{sec}$. Each point was tested twice, with an interval of 2 minutes, to express PPT as an average.

Enrolled subjects were treated with focused shock wave therapy once a week for three consecutive weeks, by a physical medicine and rehabilitation specialized physician with more than five years of expertise, using a device powered by a piezoelectric generator (PIEZOSON 100PLUS, Richard Wolf ${ }^{\circledR}$ ). At each treatment session, with the patients lying in prone decu- 
bitus position, the enthesis of the plantar fascia was clinically targeted and treated with a perpendicular technique (no ultrasound guide was used), delivering 1700 pulses (frequency = $4 \mathrm{~Hz}$ ) of an average energy flux density of $0.15 \mathrm{~mJ} / \mathrm{mm}^{2}$. During the first treatment session, the energy flux density was gradually increased from 0.05 to $0.15 \mathrm{~mJ} / \mathrm{mm}^{2}$. Gastrocnemius-soleus trigger points were treated at each session in the study group only, delivering 400 pulses (frequency $=8 \mathrm{~Hz}$ ) of an energy flux density of $0.15 \mathrm{~mJ} / \mathrm{mm}^{2}$ with the same technique. We placed a coupling gel between the probe and the skin.

\section{Statistical analysis}

Sample size considerations: considering a mean score of FFI-A equal to 5.0 before treatment and a mean score equal to 3.3 after treatment in the experimental group, a number of 25 patients per group will provide a power equal to $80 \%$ in detecting such difference, with an alpha error of 0.05 .

Quantitative variables were described as mean and standard deviation if normally distributed, as median and interquartile range if not normally distributed; qualitative ones as counts and percentages. Univariate comparisons between two groups were performed with Student t test (or with the analogous non-parametric Mann-Whitney test) for quantitative variables; chi-squared test or Fisher's exact test were used to evaluate statistical associations between qualitative variables. Univariate and multivariate linear regression models for repeated data over time, with interaction between time and treatment, were used in order to compare the score reported at FFI-A, FFI-B, PPT and Delta-PPT between the two treatment groups at the two time points. Opportune adjustment for the Delta-PPT baseline values were provided in the regression model for Delta-PPT variation in time between the two groups, since a statistically significant difference in DeltaPPT values at baseline was observed. Univariate and multivariate linear regression models for repeated data over time were performed within each group of treatment in order to compare the scores reported at FFI-A, FFI-B, PPT and DeltaPPT at different assessments. All the tests were two-sided. The significance level was set at alpha $=0.05$. Data analysis were performed with the STATA statistical software version 14 (Stata Corporation, College Station, 2015, Texas, USA).

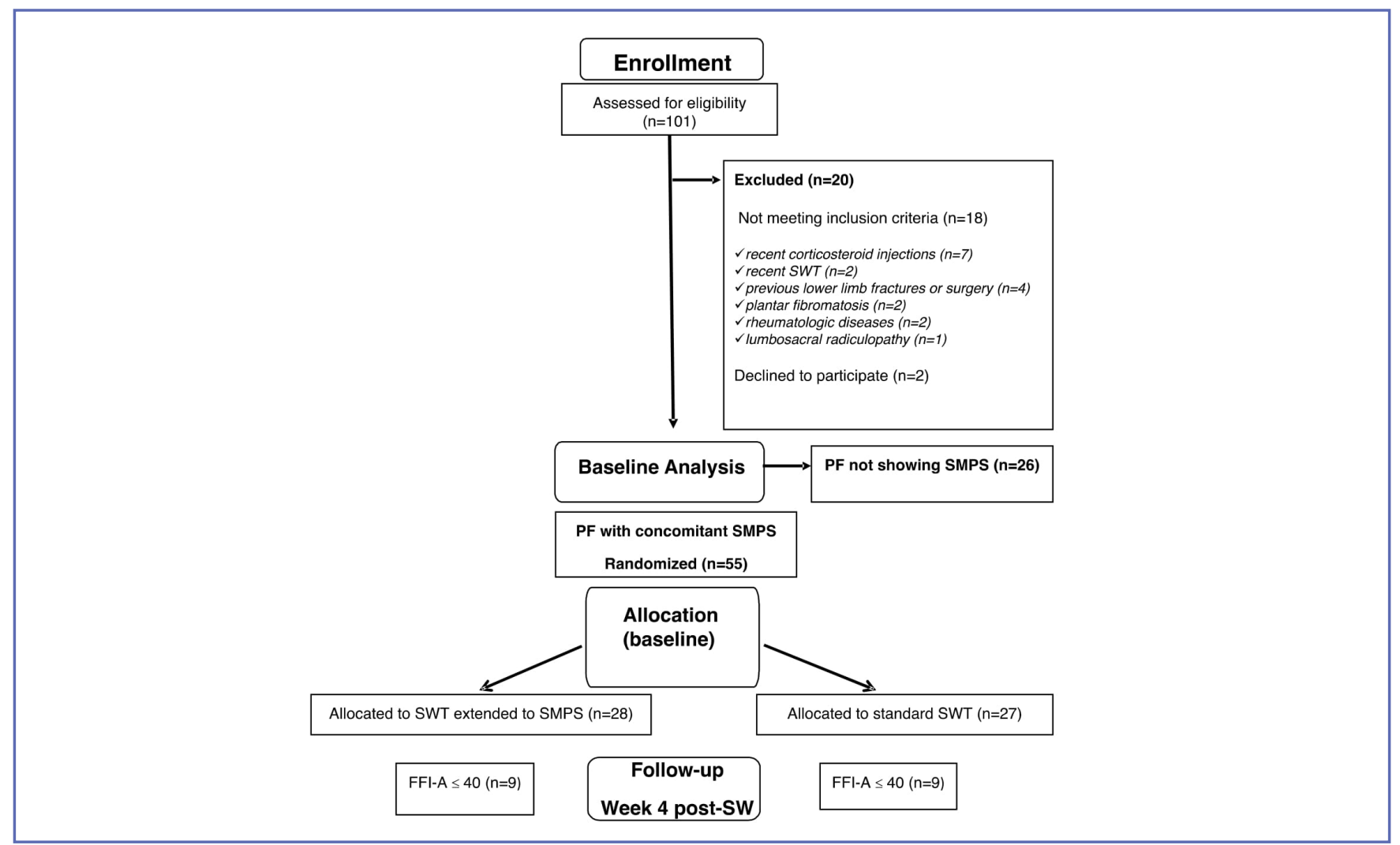

Figure 2. The Consort flow diagram of the study.

PF: plantar fasciitis; SMPS: sural myofascial pain syndrome; SWT: shock wave therapy; FFI-A: foot function indexsection A (pain). 


\section{RESULTS}

The trial profile is synthetized in the Consort flow diagram (figure 2). In the sample screened, among eighty-one subjects affected by plantar fasciitis, we enrolled $55(67.9 \%)$ patients also showing a concomitant SMPS. Data about demographics, clinical features and outcome measures of enrolled subjects at baseline are shown in table I. We observed no statistical differences regarding basic demographics, heel pain (FFI-A) and foot function (FFI-B) between the randomized patients and the non-randomized subgroup of 26 subjects satisfying eligibility criteria except for the lack of the SMPS (data not shown). Among gastrocnemius-soleus trigger points, a marked prevalence of $\operatorname{Tr} \mathrm{P} 2$ (lateral gastrocnemius) was observed (31 subjects, 56.4\%). At baseline, 58,2\% (32 cases) of the enrolled patients reported to suffer from sural myalgia, especially from night cramps (21 subjects, $38,2 \%$ ), but no statistical association between symptoms and trigger points location was found. We noticed, at baseline, no statistical differences between the study group and the control group, except for Delta-PPT values and for the distribution of TrP1 (medial gastrocnemius). All the participants felt focused shock wave therapy unpleasant but tolerable.
Outcome measures of the two groups at different observation times are graphically shown in figures 3,4 , where statistically significant $\mathrm{p}$-values for comparisons within-group are reported, respectively for FFI-A, FFI-B and for PPT and Delta-PPT. At the follow-up assessment, no statistical significance in the comparisons between groups was found for FFI-A and FFI-B total score and also for PPT and DeltaPPT values, despite comparisons within the study group (follow-up versus baseline) showed a statistically significant reduction of FFI-A total score ( $\mathrm{p}<0.001)$, FFI-B total score $(\mathrm{p}=0.029)$ and Delta-PPT values $(\mathrm{p}=0.018)$, with a consensual increase of PPT values $(p=0.017)$. We also registered an equal rate of "early responders" to treatment (FFI-A score $\leq 40$ ) in the two groups (9 subjects each; $32 \%$ ).

\section{DISCUSSION}

Our findings support the following closing remarks. We pointed out a high prevalence of sural trigger points in subjects affected by plantar fasciitis. The increase of PPT values, observed in the within study-group analysis, highlighted a quick response of the sural myofascial pain to

Table I. Demographic, clinical features and outcome measures at baseline.

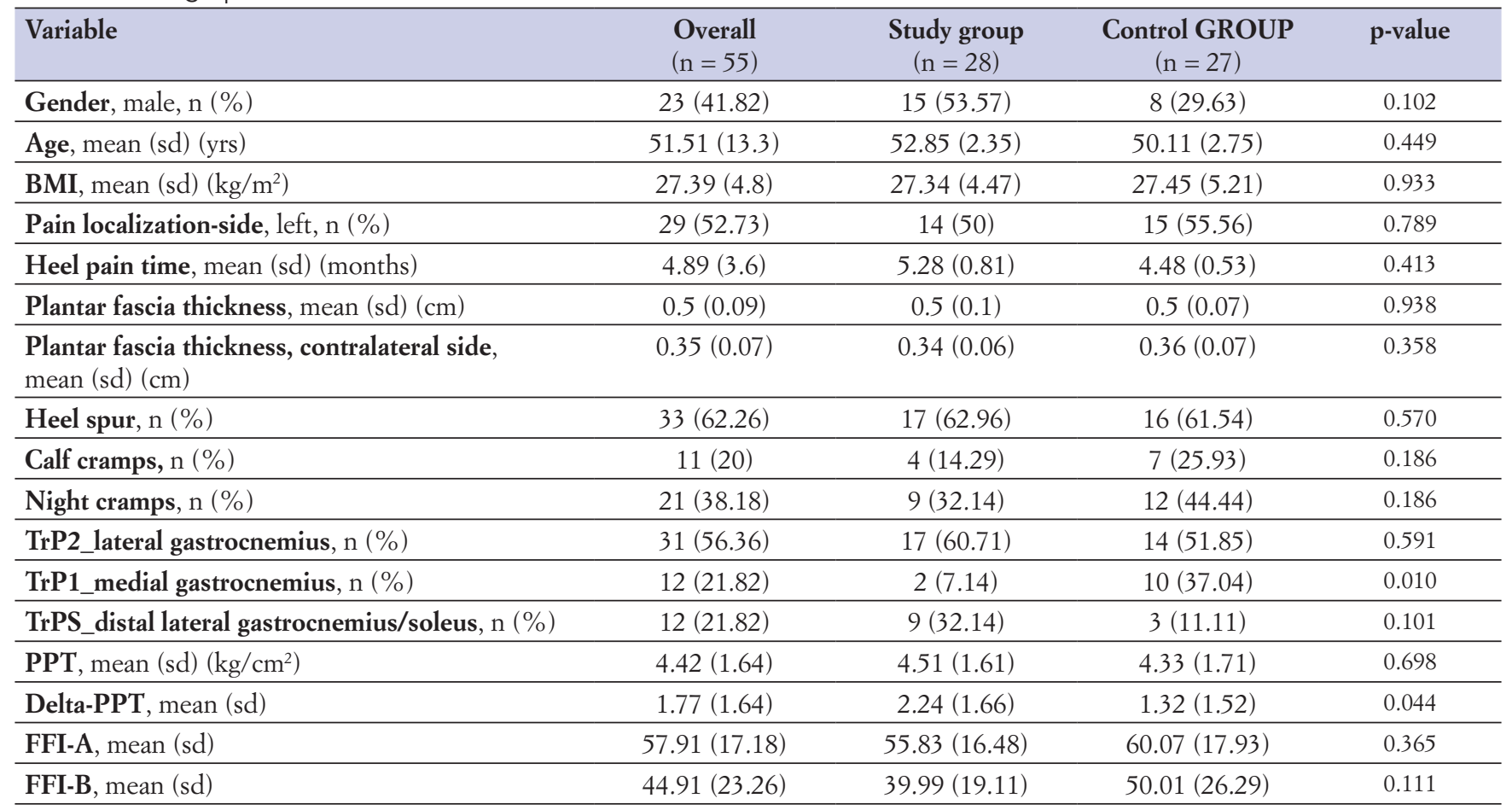

BMI: body mass index; TrP: trigger point; PPT: pressure pain threshold; Delta-PPT: delta between trigger points' PPT (on painful side) and PPT on the corresponding contralateral calf (asymptomatic side); FFI-A: foot function index- section A (pain); FFI-B: foot function index- section B (function); sd: standard deviation. A p value $<0.05$ stands for a statistically significant comparison between the two groups. 

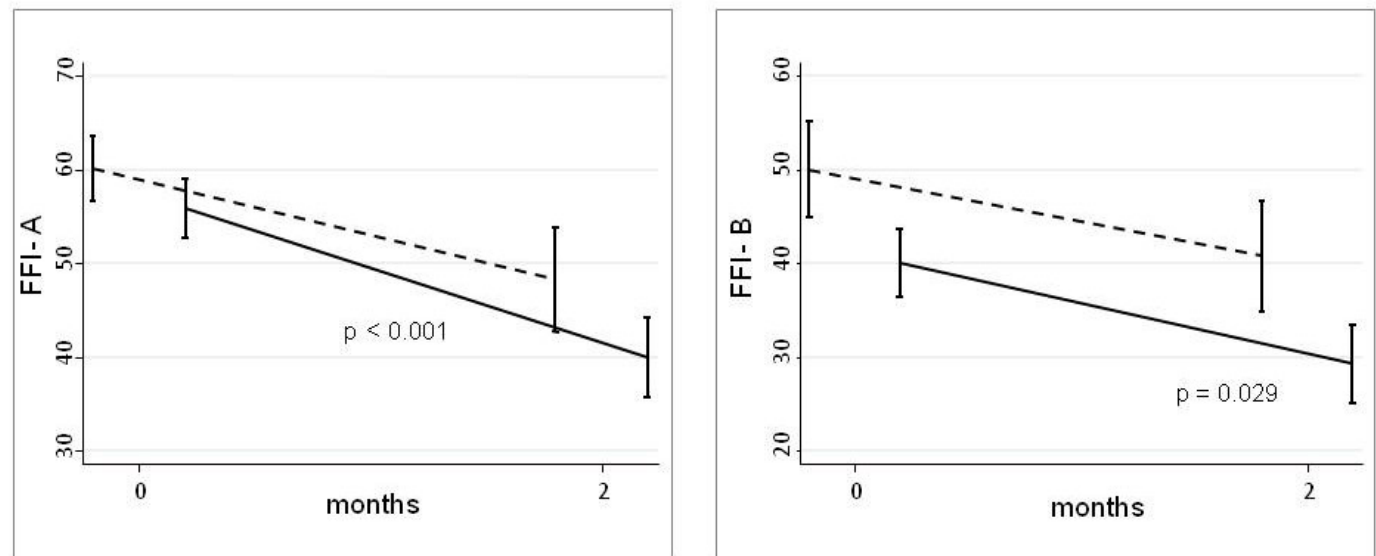

\section{---- Control group _ - Study group}

$\longmapsto \operatorname{SEM}$ (Standard Error of the Mean)

$p$ values for the comparisons within the study group overtime are reported

Figure 3. Time series plot: within group comparisons over time for FFI-A and FFI-B values.
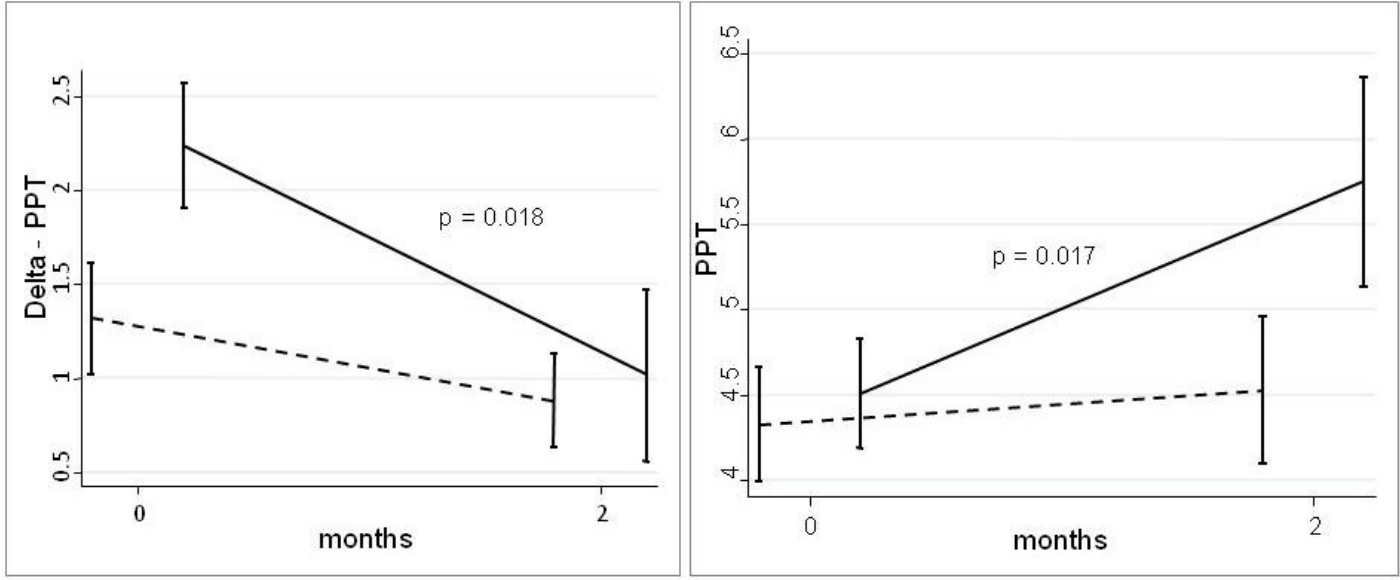

---.- Control group

Study group

$\longmapsto$ SEM (Standard Error of the Mean)

$p$ values for the comparisons within the study group overtime are reported

Figure 4. Time series plot: within group comparisons over time for Delta-PPT and PPT. 
shock waves, but insufficient in speeding up the resolution of the concomitant heel pain. Therefore, shock wave therapy extended to the gastrocnemius-soleus trigger points resulted to be ineffective, in the short-term, in reducing heel pain and sural myofascial pain, if compared to a standard treatment exclusively targeted at the plantar fascia.

Myofascial pain syndrome is basically a clinical diagnosis, the pathophysiology still being not completely understood. According to the original integrated hypothesis of trigger points' genesis, local sensitivity to pressure on a trigger point is likely to be caused by the release of inflammation mediators, in a setting of local ischemia, during prolonged myofascial contraction (1). According to the classical theory of motor adaptation to pain, the accumulation of inflammatory agents in this kind of biological setting, in turn, would sustain pain in a kind of "vicious cycle" $(20,21)$.

The more established type of medical shock wave involves focused shock wave therapy, that is a system built to deliver mechanical energy in a small focal area at a settled depth in the subcutaneous tissues (22). On the basis of the known biological effect of mechano-transduction $(23,24)$, it was postulated that shockwaves may increase perfusion, promote angiogenesis and alter the pain signalling in ischemic tissues also in case of myofascial pain syndrome $(11,12)$. A previous report also demonstrated that free nerve endings degenerate after shock wave application together to a transient dysfunction of nerve excitability at the neuromuscular junction (25). Thanks to new acquisitions in sonographic morphology of the triceps surae $(26,27)$, a recent study about gastrocnemius post-stroke spasticity highlighted a shockwave-related improvement of sonographic measures (muscle thickness, muscle fascicle length, Achilles tendon length and pennation angle) associated to a reduction of spasticity, this positive outcome probably being the direct effect of shock waves on the fibrosis of hypertonic muscles (28).

Nowadays extracorporeal shock wave therapy should be considered an emerging treatment for myofascial pain, whose classical treatment implies various conservative approaches such as dry needling, spray and stretch and pharmacological treatment (12). Muller-Ehrenberg first reported a reduction in myofascial pain after three months in a sample of thirty subjects treated with focused shock waves (10). Moghtaderi showed that treating with shock waves both the plantar fascia and the gastrocnemius-soleus trigger points is more effective in the mid-term (two months after the end of treatment) in reducing chronic heel pain than solely treating the plantar fascia (13). A previous report by Jeon highlighted that a focused shock wave therapy is effective in reducing myofascial pain of the trapezius muscle in a short-term perspective (at the end of treatment in this case) (11). This result suggests an earlier response to shock wave therapy in myofascial pain syndromes than in tendinop- athies, wherein, according to the literature, two-three months are usually needed to obtain an improvement of tendon pain $(8,29,30)$. Despite we also noticed a trend of increase of PPT values in the within study-group analysis, in the present trial the extension of the shock wave treatment to sural trigger points seems to bring no significant advantages in the shortterm (one month after the end of treatment).

The majority of studies about shock wave therapy in myofascial pain syndromes made use of focused devices $(10,11,13)$. Ramon suggested the inclusion of radial shock waves in a protocol of focalized shock wave therapy for myofascial pain and fibromyalgia (12), but no definitive comparison of efficacy (between radial and shock wave therapy) is actually available for myofascial pain. For what regards ancillary treatment strategies, Cinar showed that the use of stretching exercises for the posterior chain and for plantar fascia provide an improvement in heel pain in patients affected by plantar fasciitis (31). More recently, Notarnicola reported better results in terms of recovery of dorsal and plantar flexion applying a shock wave treatment targeted to the plantar fascia and to the gastrocnemius muscle (32).

The use of algometry associated to clinical findings helped us to better monitor trigger points response to therapy. Baseline data from the present study confirm that a difference in PPT measurement (Delta-PPT) exceeding $2 \mathrm{Kg} / \mathrm{cm}^{2}$ between the painful and the normal side could be a helpful reference to confirm the diagnosis of SMPS, as proposed by Fischer in case of myofascial pain (2). Regarding algometric assessment in clinical practice, we propose for future studies to measure PPT on the asymptomatic side using a painless site in the upper limb, in order to reduce possible influences of an altered weight distribution between the lower limbs secondary to unilateral chronic heel pain.

Our findings should be read in light of the following limitations. Patients could not be blinded to the group assignment, but the influence of their expectations about the outcome was probably marginal, since both groups showed improvement over time. Our follow-up failed to assess the outcome of the treatment in mid-term and long-term perspectives. However, results reported by Moghtaderi showed that treating with shock waves both the plantar fascia and the gastrocnemius-soleus trigger points is more effective in the mid-term in reducing chronic heel pain than solely treating the plantar fascia (11) and results from previous clinical studies on various tendinopathies showed that a clear improvement of symptoms was usually maintained at the one-year follow-up, when achieved three to twelve weeks after a focused shock wave treatment $(24,25)$.

Further studies, with a larger sample population and with longer follow-up periods, are needed to clarify how shock waves may affect myofascial syndromes associated to tendinopathies. 


\section{CONFLICT OF INTERESTS}

The authors declare that they have no conflict of interests.

\section{REFERENCES}

1. Travell J, Simons DG. Myofascial Pain and Dysfunction. The Trigger Point Manual. Volume 2. Philadelphia: Lippincott Williams \& Wilkins; 1993.

2. Fischer AA. Documentation of myofascial trigger points. Arch Phys Med Rehabil 1988;69(4):286-91.

3. Buchbinder R. Clinical practice. Plantar fasciitis. N Engl J Med 2004:350(21):2159-66.

4. Thomas JL, Christensen JC, Kravitz SR, et al. American College of Foot and Ankle Surgeons heel pain committee. The diagnosis and treatment of heel pain: a clinical practice guideline-revision 2010. J Foot Ankle Surg 2010;49(3 Suppl):S1-19.

5. Petraglia F, Ramazzina I, Costantino C. Plantar fasciitis in athletes: diagnostic and treatment strategies. A systematic review. Muscle Ligaments Tendons J 2017;7(1):107-18.

6. Patel A, DiGiovanni B. Association between plantar fasciitis and isolated contracture of the gastrocnemius. Foot Ankle Int 2011:32:5-8.

7. Bolivar YA, Munuera PV, Padillo JP. Relationship between tightness of the posterior muscles of the lower limb and plantar fasciitis. Foot Ankle Int 2013;34:42-8.

8. Gollwitzer H, Saxena A, DiDomenico LA, et al. Clinically relevant effectiveness of focused extracorporeal shock wave therapy in the treatment of chronic plantar fasciitis: a randomized, controlled multicenter study. J Bone Joint Surg Am 2015;97(9):701-8.

9. Sun J, Gao F, Wang Y, Sun W, Jiang B, Li Z. Extracorporeal shock wave therapy is effective in treating chronic plantar fasciitis: A meta-analysis of RCTs. Medicine (Baltimore) 2017;96(15):e6621.

10. Muller-Ehrenberg H, Licht G. Diagnosis and therapy of myofascial pain syndrome with focused shock waves. Medizinisch Orthopadische Technik 2005;5:1-6.

11. Jeon JH, Jung YJ, Lee JY, et al. The effect of extracorporeal shock wave therapy on myofascial pain syndrome. Ann Rehabil Med 2012;36(5):665-74.

12. Ramon S, Gleitz M, Hernandez L, Romero LD. Update on the efficacy of extracorporeal shockwave treatment for myofascial pain syndrome and fibromyalgia. Int J Surg 2015;24(Pt B):201-6.

13. Moghtaderi A, Khosrawi S, Dehghan F. Extracorporeal shock wave therapy of gastroc-soleus trigger points in patients with plantar fasciitis: A randomized, placebo-controlled trial. Adv Biomed Res 2014:3:99.

14. Padulo J, Oliva F, Frizziero A, Maffulli N. Muscles, Ligaments and Tendons Journal- Basic principles and recommendations in clinical and field Science Research:2018 update. Muscles Ligaments Tendons J 2018;8(3):305-7

15. Martinelli N, Scotto GM, Sartorelli E, Bonifacini C, Bianchi A, Malerba F. Reliability, validity and responsiveness of the Italian version of the Foot Function Index in patients with foot and ankle diseases. Qual Life Res 2014;23(1):277-84.

16. Polianskis R, Graven-Nielsen T, Arendt-Nielsen L. Computer-controlled pneumatic pressure algometry-a new technique for quantitative sensory testing. Eur J Pain 2001;5(3):267-77.

17. Karabay N, Toros T, Hurel C. Ultrasonographic evaluation in plantar fasciitis. J Foot Ankle Surg 2007;46(6):442-6.

18. Mohseni-Bandpei MA, Nakhaee M, Mousavi ME, Shakourirad A, Safari MR, Vahab Kashani R. Application of ultrasound in the assessment of plantar fascia in patients with plantar fasciitis: a systematic review. Ultrasound Med Biol 2014;40(8):1737-54.

19. Park JW, Yoon K, Chun KS, et al. Long-term outcome of low-energy extracorporeal shock wave therapy for plantar fasciitis: comparative analysis according to ultrasonographic findings. Ann Rehabil Med 2014;38(4):534-40.

20. Hodges PW, Tucker K. Moving differently in pain: a new theory to explain the adaptation to pain. Pain 2011;152(3 Suppl):S90-8.

21. Roland MO. A critical review of the evidence for a pain-spasmpain cycle in spinal disorders. Clin Biomech (Bristol, Avon) 1986;1(2):102-9.

22. Ogden JA, Toth-Kischkat A, Schultheiss R. Principles of shock wave therapy. Clin Orthop Relat Res 2001;(387):8-17.

23. Ioppolo F, Rompe JD, Furia J, Cacchio A. Clinical application of shock wave therapy (SWT) in musculoskeletal disorders. Eur J Phys Rehabil Med 2014;50(2):217-30.

24. D'Agostino MC, Craig K, Tibalt E, Respizzi S. Shock wave as biological therapeutic tool: From mechanical stimulation to recovery and healing, through mechanotransduction. Int J Surg 2015;24(Pt B):147-53.

25. HausdorfJ, Lemmens MA, Heck KD, et al. Selective loss of unmyelinated nerve fibers after extracorporeal shockwave application to the musculoskeletal system. Neuroscience 2008;155(1):138-44.

26. Chow RS, Medri MK, Martin DC, Leekam RN, Agur AM, McKee NH. Sonographic studies of human soleus and gastrocnemius muscle architecture: gender variability. Eur J Appl Physiol 2000;82:236-244.

27. Lima K, Martins N, Pereira W, Oliveira L. Triceps surae elasticity modulus measured by shear wave elastography is not correlated to the plantar flexion torque. Muscle Ligaments Tendons J 2017;7(2):347-52.

28. Lee $\mathrm{CH}$, Lee SU, Lee SH, Yoo JI. Ultrasonographic evaluation for the effect of extracorporeal shock wave therapy on gastrocnemius muscle spasticity in patients with chronic stroke. PM R 2019;11(4):363-71.

29. Gerdesmeyer L, Wagenpfeil S, Haake M, et al. Extracorporeal shock wave therapy for the treatment of chronic calcifying tendonitis of the rotator cuff: a randomized controlled trial. JAMA 2003;290(19):2573-80.

30. Lee SY, Cheng B, Grimmer-Somers K. The midterm effectiveness of extracorporeal shockwave therapy in the management of chronic calcific shoulder tendinitis. J Shoulder Elbow Surg 2011;20(5):845-54.

31. Cinar E, Saxena S, Uygur F. Combination therapy versus exercise and orthotic support in the management of pain in plantar fasciitis: a randomized controlled trial. Foot Ankle Int 2018;39(4)-406-14.

32. Notarnicola $A$, Maccagnano $G$, Farì $G$ et al. Extracorporeal shockwave therapy for plantar fasciitis and gastrocnemius muscle: effectiveness of a combined treatment. J Biol Regul Homeost Agents 2020;34(1):285-90. 